\title{
Detection of icing and actuators faults in the longitudinal dynamics of small UAVs using an LPV proportional integral unknown input observer
}

\author{
Damiano Rotondo, Andrea Cristofaro, Tor Arne Johansen, Fatiha Nejjari and Vicenç Puig
}

\begin{abstract}
This paper proposes a linear parameter varying proportional integral unknown input observer (PI-UIO) for the diagnosis of actuator faults and icing in unmanned aerial vehicles. It is shown that in presence of sensor noise, the proposed PI-UIO has the advantage of being affected by the noise, but not by its derivative. Another contribution of the paper is the introduction of an icing to wind/noise ratio, which allows performing an optimal tuning of some PI-UIO design parameters. Results obtained with a Zagi Flying Wing simulator are used to validate the effectiveness of the proposed approach.
\end{abstract}

\section{INTRODUCTION}

The problem of fault detection and isolation is the problem of generating diagnostic signals sensitive to the occurrence of faults [1]. In recent years, this problem has attracted a lot of attention, and several approaches have been proposed as possible solutions, e.g. the geometric [1], [2] and the observer-based [3], [4] ones.

Among the most significant faults that affect aviation safety, there is icing, i.e. the accretion of ice on aircraft wings and control surfaces [5]. By decreasing the lift and increasing the drag and the mass of the vehicle, icing has a profound impact on the aircraft performance, inducing a safety risk that can lead potentially to crashing [6]. When an aircraft flies through clouds at an ambient temperature below freezing, supercooled water droplets (SWD) suspended in the cloud can impact the aircraft surface and freeze immediately, thus accreting the ice [7]. The rate and the severity of icing are determined by several factors, such as shape and roughness of the impacting surface, vehicle speed, air temperature and relative humidity [8].

This work has been supported by a grant from Iceland, Liechtenstein and Norway through the EEA Financial Mechanism. Operated by Universidad Complutense de Madrid (ref. 006-ABEL-IM-2014B). The authors also acknowledge support by the Spanish Government (Ministerio de Economía y Competividad) and FEDER through the projects CICYT ECOCIS (ref. DPI2013-48243-C2-1-R) and CICYT HARCRICS (ref. DPI2014-58104-R), by AGAUR through the contract FI-DGR 2014 (ref. 2014FI_B1_00172), by the DGR of the Generalitat de Catalunya (ref. 2014/SGR/374) and by the Research Council of Norway through the Centres of Excellence funding scheme (ref. 223254 - NTNU AMOS). D. Rotondo and A. Cristofaro are also supported by the ERCIM Alain Bensoussan Fellowship programme.

D. Rotondo, F. Nejjari and V. Puig are with Advanced Control Systems Group (SAC), Universitat Politècnica de Catalunya (UPC), TR11, Rambla de Sant Nebridi, 10, 08222 Terrassa, Spain. V. Puig is also with Institut de Robotica i Informatica Industrial (IRI), UPC-CSIC, Carrer de Llorens i Artigas, 4-6, 08028 Barcelona, Spain. D. Rotondo, A Cristofaro and T. A. Johansen are with Centre for Autonomous Marine Operations and Systems (NTNU AMOS), Department of Engineering Cybernetics, Norwegian University of Science and Technology, Norway. A Cristofaro is also with School of Science and Technology, University of Camerino, via Madonna delle Carceri, 62302, Camerino, Italy. e-mail : damiano (dot) rotondo (at) yahoo (dot) it
Recently, some anti- and de-icing systems for unmanned aerial vehicles (UAVs) have been proposed. For example, layers of coating material containing carbon nanotubes, that can be heated up quickly using an onboard energy source, have been used to paint the wing surfaces [9]. However, due to the large power consumption, fault/icing detection schemes [10] with fast and accurate responses are needed for assuring high efficiency. The approaches recently applied to icing detection in aircrafts and UAVs include unknown input observers (UIOs) [11]-[13], adaptive multiple models [14] and statistical methods [15]. Furthermore, a linear parameter varying (LPV) UIO-based icing detection scheme has been presented in [16], with the main advantage of being consistent with the aircraft dynamics for a wide range of operating conditions.

It is known that when noise affects the sensor measurements, proportional integral (PI) observers achieve better convergence and filtering properties [17]. For this reason, the study of PI-UIOs has attracted some interest, as testified by the number of articles dealing with this subject, e.g. [18]-[20]. The goal of this paper is to improve the results obtained in [16], where the considered LPV UIO had only a proportional action. It is shown that when the sensor noise is included in the problem formulation, the estimation error dynamics obtained with [16] is affected by the noise derivative, which has a strong effect on the achievable performances, due to the high-frequency content of the noise signal. Even though the noise could be dealt with using low-pass filters, the optimal design and tuning of such filters would be a challenging problem, which motivates to seek alternative solutions for dealing with the noise within the observer design. For this reason, in order to overcome the limitation of the proportional UIO, an LPV PI-UIO is proposed. Moreover, another contribution of this work with respect to [16] is the introduction of an icing to wind/noise ratio (IWNR), which allows performing an optimal choice of some PI-UIO design parameters.

The paper is structured as follows. Section II presents the quasi-LPV model of the UAV subject to icing and faults. Section III is dedicated to the design of an LPV PI-UIO for the actuator fault and icing diagnosis. Section IV validates the effectiveness of the proposed approach using a Zagi Flying Wing simulator. Finally, Section V outlines the main conclusions and suggests some future work. 


\section{QUASI-LPV MODEL OF THE UAV SUBJECT TO ICING/FAULTS}

Following [16], the longitudinal equations of motion of an aircraft [21], under low-angle-of-attack condition, can be brought to a quasi-LPV form using the nonlinear embedding in the parameters approach [22], [23], as follows ${ }^{1}$ (see Table I for a description of the symbols):

$$
\dot{x}=A(u, w, q, \theta) x+B(u, w) v+d(\theta)
$$

where $x=(u, w, q, \theta)^{T}$ is the state vector, $v=\left(\delta_{t}^{2}, \delta_{e}\right)^{T}$ is the input vector, and the matrices $A(u, w, q, \theta), B(u, w), d(\theta)$ are given by:

$$
\begin{gathered}
A(u, w, q, \theta)=\left(\begin{array}{cccc}
a_{11}(\cdot) & a_{12}(\cdot) & a_{13}(\cdot) & 0 \\
a_{21}(\cdot) & a_{22}(\cdot) & a_{23}(\cdot) & 0 \\
a_{31}(\cdot) & a_{32}(\cdot) & a_{33}(\cdot) & 0 \\
0 & 0 & 1 & 0
\end{array}\right) \\
B(u, w)=\left(\begin{array}{cc}
b_{11} & b_{12}(\cdot) \\
0 & b_{22}(\cdot) \\
0 & b_{32}(\cdot) \\
0 & 0
\end{array}\right)
\end{gathered}
$$

where:

$$
\begin{aligned}
& a_{11}(\cdot)=\frac{\rho u S}{2 m}\left[\left(C_{L_{0}}+C_{L_{\alpha}} \alpha\right) \sin \alpha-\left(C_{D_{0}}+C_{D_{\alpha}} \alpha\right) \cos \alpha-\frac{S_{\text {prop }} C_{\text {prop }}}{S}\right] \\
& a_{12}(\cdot)=\frac{\rho w S}{2 m}\left[\left(C_{L_{0}}+C_{L_{\alpha}} \alpha\right) \sin \alpha-\left(C_{D_{0}}+C_{D_{\alpha}} \alpha\right) \cos \alpha-\frac{S_{\text {prop }} C_{\text {prop }}}{S}\right] \\
& a_{13}(\cdot)=-w+\frac{\rho S c V_{a}}{4 m}\left(C_{L_{q}} \sin \alpha-C_{D_{q}} \cos \alpha\right) \\
& a_{21}(\cdot)=-\frac{\rho u S}{2 m}\left[\left(C_{D_{0}}+C_{D_{\alpha}} \alpha\right) \sin \alpha+\left(C_{L_{0}}+C_{L_{\alpha}} \alpha\right) \cos \alpha\right] \\
& a_{22}(\cdot)=-\frac{\rho w S}{2 m}\left[\left(C_{D_{0}}+C_{D_{\alpha}} \alpha\right) \sin \alpha+\left(C_{L_{0}}+C_{L_{\alpha}} \alpha\right) \cos \alpha\right] \\
& a_{23}(\cdot)=u-\frac{\rho S c V_{a}}{4 m}\left(C_{D_{q}} \sin \alpha+C_{L_{q}} \cos \alpha\right) \\
& a_{31}(\cdot)=\frac{\rho S c u}{2 J_{y}}\left(C_{m_{0}}+C_{m_{\alpha}} \alpha\right) \\
& a_{32}(\cdot)=\frac{\rho S c w}{2 J_{y}}\left(C_{m_{0}}+C_{m_{\alpha}} \alpha\right) \\
& a_{33}(\cdot)=\frac{\rho V_{a} S c^{2}}{4 J_{y}} C_{m_{q}} \\
& b_{11}=\frac{\rho S_{p r o p} C_{p r o p}}{2 m} k_{m}^{2} \\
& b_{12}(\cdot)=\frac{\rho S V_{a}^{2}}{2 m}\left(C_{L_{\delta_{e}}} \sin \alpha-C_{D_{\delta_{e}}} \cos \alpha\right) \\
& b_{22}(\cdot)=-\frac{\rho V_{a}^{2} S}{2 m}\left(C_{D_{\delta_{e}}} \sin \alpha+C_{L_{\delta_{e}}} \cos \alpha\right) \\
& b_{32}(\cdot)=\frac{\rho V_{a}^{2} S c}{2 J_{y}} C_{m_{\delta_{e}}}
\end{aligned}
$$

Remark: It is worth highlighting that although (1) is in a linear form, it is an equivalent representation of the nonlinear equations that describe the longitudinal equations of motion of an aircraft (no linearization is performed).

\footnotetext{
${ }^{1}$ To ease the notation, the dependence of variables on time $t$ is omitted.
}

TABLE I

SYMBOLS DESCRIPTION

\begin{tabular}{cc}
\hline Symbol & Description \\
\hline$u$ & Horizontal velocity relative to the wind \\
$w$ & Vertical velocity relative to the wind \\
$q$ & Pitch rate \\
$\theta$ & Pitch angle \\
$g$ & Gravitational acceleration \\
$\rho$ & Air density \\
$S$ & Wing surface area \\
$m$ & Airframe mass \\
$\alpha$ & Angle-of-attack \\
$c$ & Mean aerodynamic chord of the wing \\
$S_{\text {prop }}$ & Area of the propeller \\
$k_{m}$ & Efficiency of the motor \\
$J_{y}$ & Element of the inertia matrix \\
$V_{a}$ & Airspeed with respect to the mass \\
$\delta_{t}$ & Throttle deflection \\
$C_{i}$ & Stability and control derivatives \\
\hline
\end{tabular}

\section{A. Unknown inputs acting on the aircraft}

The aircraft may be affected by undesired effects, such as wind, actuator faults and accretion of ice on its surfaces (icing).

More specifically, the wind effect is modeled as an additive disturbance vector $\mathcal{W}$ given by:

$\mathcal{W}=\left(\begin{array}{cc}-\cos \theta & -\sin \theta \\ -\sin \theta & \cos \theta \\ 0 & 0 \\ 0 & 0\end{array}\right)\left(\begin{array}{c}\dot{\omega}_{x} \\ \dot{\omega}_{z}\end{array}\right)=H_{1}(\theta) \dot{\omega}_{x}+H_{2}(\theta) \dot{\omega}_{z}$

where $\dot{\omega}_{x}$ and $\dot{\omega}_{z}$ are the wind accelerations in the horizontal and vertical direction in the inertial frame, respectively. The Dryden model [24], which uses spatially varying stochastic processes with specified power spectral densities, is used to represent the components of the wind gusts in a realistic way.

Actuator faults are represented as an unknown input term $\mathcal{F}$ given by:

$$
\mathcal{F}=\left(\begin{array}{cc}
b_{11} & b_{12}(\cdot) \\
0 & b_{22}(\cdot) \\
0 & b_{32}(\cdot) \\
0 & 0
\end{array}\right)\left(\begin{array}{l}
\varphi_{t} \\
\varphi_{e}
\end{array}\right)=B_{1} \varphi_{t}+B_{2}(u, w) \varphi_{e}
$$

where $\varphi_{t}$ and $\varphi_{e}$ correspond to faults in propellers/motors and elevator, respectively.

Finally, the icing effect is modeled as an additive timedependent disturbance $\mathcal{E}(u, w, q) \eta$, where $\eta$ is the icing severity factor [25], and $\mathcal{E}(u, w, q)$ is given by [16]:

$$
\mathcal{E}(u, w, q)=\left(\begin{array}{llll}
\mathcal{E}_{1}(u, w, q) & \mathcal{E}_{2}(u, w, q) & \mathcal{E}_{3}(u, w, q) & 0
\end{array}\right)^{T}
$$

with:

$$
\begin{aligned}
\mathcal{E}_{1}(u, w, q) & =\frac{\rho V_{a}^{2} S}{2 m}\left[\left(K_{L_{0}} C_{L_{0}}+K_{L_{\alpha}} C_{L_{\alpha}} \alpha\right) \sin \alpha\right. \\
& -\left(K_{D_{0}} C_{D_{0}}+K_{D_{\alpha}} C_{D_{\alpha}} \alpha\right) \cos \alpha \\
& +\left(K_{L_{q}} C_{L_{q}} \sin \alpha-K_{D_{q}} C_{D_{q}} \cos \alpha\right) \frac{c q}{2 V_{a}} \\
& \left.+\left(K_{L_{\delta_{e}}} C_{L_{\delta_{e}}} \sin \alpha-K_{D_{\delta_{e}}} C_{D_{\delta_{e}}} \cos \alpha\right) \delta_{e}\right]
\end{aligned}
$$




$$
\begin{aligned}
\mathcal{E}_{2}(u, w, q) & =-\frac{\rho V_{a}^{2} S}{2 m}\left[\left(K_{D_{0}} C_{D_{0}}+K_{D_{\alpha}} C_{D_{\alpha}} \alpha\right) \sin \alpha\right. \\
& +\left(K_{L_{0}} C_{L_{0}}+K_{L_{\alpha}} C_{L_{\alpha}} \alpha\right) \cos \alpha \\
& +\left(K_{D_{q}} C_{D_{q}} \sin \alpha+K_{L_{q}} C_{L_{q}} \cos \alpha\right) \frac{c q}{2 V_{a}} \\
& \left.+\left(K_{D_{\delta_{e}}} C_{D_{\delta_{e}}} \sin \alpha+K_{L_{\delta_{e}}} C_{L_{\delta_{e}}} \cos \alpha\right) \delta_{e}\right] \\
\mathcal{E}_{3}(u, w, q) & =\frac{\rho V_{a}^{2} S c}{2 J_{y}}\left(K_{m_{0}} C_{m_{0}}+K_{m_{\alpha}} C_{m_{\alpha}} \alpha\right. \\
& \left.+K_{m_{q}} C_{m_{q}} \frac{c q}{2 V_{a}}+K_{m_{\delta_{e}}} C_{m_{\delta_{e}}} \delta_{e}\right)
\end{aligned}
$$

where the coefficients $K_{i}$ depend on aircraft design and operating conditions [25].

The undesired effects are added as additive terms (unknown inputs) in the LPV state equation (1), modifying it as follows:

$$
\dot{x}=A(u, w, q, \theta) x+B(u, w) v+B_{u n}(u, w, q, \theta) v_{u n}+d(\theta)
$$

where $v_{u n}=\left(\dot{\omega}_{x}, \dot{\omega}_{z}, \varphi_{t}, \varphi_{e}, \eta\right)^{T}$ is the unknown input vector, and the matrix $B_{u n}(u, w, q, \theta)$ is given by:

$B_{u n}(u, w, q, \theta)=\left(\begin{array}{lllll}H_{1}(\theta) & H_{2}(\theta) & B_{1} & B_{2}(u, w) & E(u, w, q)\end{array}\right)$

Assuming that the UAV is equipped with airspeed measurement device (pitot tube), GPS and inertial sensors, all state variables are supposed to be available and hence the output matrix of the system verifies $C=I_{4 \times 4}$.

\section{ICING/FAULT DIAGNOSIS USING AN LPV PI-UIO}

For further reasoning, let us rewrite (10) in a more general form:

$$
\dot{x}=A(\vartheta) x+B(\vartheta) v+B_{u n}(\vartheta) \mathrm{v}_{u n}+d(\vartheta)
$$

where $\vartheta$ is some varying parameter vector, containing exogenous variables, endogenous variables (e.g. states and/or inputs), or a combination of them.

The output equation is given by:

$$
y=x+v
$$

where $v$ is the measurement noise.

It can be shown that, by using the proportional UIO proposed in [16], the dynamics of the estimation error $\varepsilon=$ $x-\hat{x}$ (with $\hat{x}$ denoting the state estimation provided by the UIO) would depend on the noise derivative $\dot{v}$, which has a strong effect on the achievable performances, due to the highfrequency content of the noise signal. In order to overcome this problem, an LPV PI-UIO is proposed, as stated in the following theorem.

Theorem 1: Let $R(\vartheta), F(\vartheta)$ and $T(\vartheta)$ be some given matrix functions, with $\operatorname{det}(R(\vartheta)) \neq 0$ and $\operatorname{det}(T(\vartheta)) \neq 0$ $\forall \vartheta \in \Theta$, where $\Theta$ is the set of possible values for $\vartheta$, and calculate the following matrix function:

$$
K(\vartheta)=T(\vartheta)^{-1}\left[A(\vartheta)+R(\vartheta)^{-1}(\dot{R}(\vartheta)-F(\vartheta) R(\vartheta))\right]
$$

where $\dot{R}(\vartheta)$ is the time derivative of $R(\vartheta)$. Then, an UIO for the system (12)-(13) is given by:

$$
\begin{aligned}
\dot{z} & =A(\vartheta) z+B(\vartheta) v+d(\vartheta)+A(\vartheta) T(\vartheta) w-\dot{T}(\vartheta) w \\
\dot{w} & =K(\vartheta)(y-\hat{x}) \\
\hat{x} & =z+T(\vartheta) w
\end{aligned}
$$

where $\dot{T}(\vartheta)$ is the time derivative of $T(\vartheta)$, with an estimation error $\varepsilon_{R}=R(\vartheta)(x-\hat{x})$ that obeys:

$$
\dot{\varepsilon}_{R}=F(\vartheta) \varepsilon_{R}+R(\vartheta) B_{u n}(\vartheta) v_{u n}-R(\vartheta) T(\vartheta) K(\vartheta) v
$$

Proof: The estimation error dynamics, taking into account (12) and (17), is given by:

$$
\begin{aligned}
& \dot{\varepsilon}_{R}=R(\vartheta)(\dot{x}-\dot{\hat{x}})+\dot{R}(\vartheta)(x-\hat{x})=R(\vartheta)[A(\vartheta) x+B(\vartheta) \vartheta \cdots \\
& \left.\cdots+B_{u n}(\vartheta) v_{u n}+d(\theta)-\dot{z}-T(\vartheta) \dot{w}-\dot{T}(\vartheta) w\right]+\dot{R}(\vartheta)(x-\hat{x})
\end{aligned}
$$

Considering now (15)-(17), (19) can be rewritten as:

$$
\begin{aligned}
\dot{\varepsilon}_{R}= & {[R(\vartheta) A(\vartheta)-R(\vartheta) T(\vartheta) K(\vartheta)+\dot{R}(\vartheta)](x-\hat{x}) \cdots } \\
& \cdots+R(\vartheta) B_{u n}(\vartheta) v_{u n}-R(\vartheta) T(\vartheta) K(\vartheta) v
\end{aligned}
$$

Then, it is easy to see that (18) results from (20) taking into account (14), which completes the proof. $\square$

Remark 1: The rotation through the matrix $R(\vartheta)$ is needed in order to obtain a term of the form $R(\vartheta) B_{u n}(\vartheta)$ in the estimation error equation (18). As shown in the following, this term is of paramount importance in order to achieve the unknown input effect decoupling.

\section{A. Unknown input effects decoupling}

The matrix function $R(\vartheta)$ can be chosen to constrain the range of the matrix function $R(\vartheta) B_{u n}(\vartheta)$, in such a way that different output directions of the residuals are assigned to wind disturbance, actuator faults and icing effects, respectively, with the aim of identifying the cause for some detected system malfunctions. Even though a complete decoupling of the wind disturbance and icing effects from the actuator faults cannot be achieved due to the superposition of effects and the lack of degrees of freedom in the UIO design, a successful fault/icing diagnosis can still be achieved.

To this aim, let us notice that as long as $\cos \theta \neq 0$, the following condition holds:

$$
\mathcal{E}(u, w, q, \theta)=\mathcal{E}(t) \in \operatorname{span}\left[\begin{array}{lll}
B_{1} & B_{2}(u, w) & H_{2}(\theta)
\end{array}\right] \forall t \geq 0
$$

Then, the target is to design the UIO matrix function $R(\vartheta)$ with the following properties:

$$
\begin{aligned}
& R(\vartheta) B_{1}=b_{11} e_{1} \\
& R(\vartheta) B_{2}(u, w)=V_{a}^{2} e_{2} \\
& R(\vartheta) H_{2}(\theta)=\cos \theta e_{3}
\end{aligned}
$$

where $e_{1}, e_{2}, e_{3}, e_{4}$ denote the canonical basis vectors of $\mathbb{R}^{4}$. As discussed in the following subsection, together with an appropriate choice of the matrix function $F(\vartheta),(22)$-(24) will assure that $\varphi_{t}, \varphi_{e}$ and $\dot{\omega}_{z}$ will affect different components of the vector $\varepsilon_{R}$, i.e. $\varepsilon_{R, 1}, \varepsilon_{R, 2}$ and $\varepsilon_{R, 3}$, respectively. 
It is easy to check that the following matrix satisfies (22)(24):

$$
\begin{aligned}
R(u, w, \theta) & =\left(\begin{array}{cccc}
1 & \tan \theta & r_{13}(u, w, \theta) & 0 \\
0 & 0 & \frac{2 J_{y}}{\rho S c C_{\delta_{e}}} & 0 \\
0 & 1 & r_{33}(u, w) & 0 \\
0 & 0 & 0 & 1
\end{array}\right) \\
r_{13}(u, w, \theta) & =\frac{J_{y}}{m c C_{m_{\delta_{e}}}}\left[\left(C_{D_{\delta_{e}}}+C_{L_{\delta_{e}}} \tan \theta\right) \cos \alpha\right. \\
& \left.+\left(C_{D_{\delta_{e}}} \tan \theta-C_{L_{\delta_{e}}}\right) \sin \alpha\right] \\
r_{33}(u, w) & =\frac{J_{y}}{m c C_{m_{\delta_{e}}}}\left(C_{L_{\delta_{e}}} \cos \alpha+C_{D_{\delta_{e}}} \sin \alpha\right)
\end{aligned}
$$

Due to the presence of $\cos \alpha$ and $\sin \alpha$ in $r_{13}(u, w, \theta)$ and $r_{33}(u, w)$, the matrix function $\dot{R}(\vartheta)$ will contain $\dot{\alpha}$, which is affected by unknown faults and icing. However, these elements can be assumed to be small in size, such that $\dot{R}(\vartheta)$ can be approximated with the following matrix, obtained from (25) assuming a constant $\alpha$ :

$$
\begin{aligned}
& \tilde{\dot{R}}(u, w, q, \theta)=\left(\begin{array}{cccc}
0 & \tilde{\dot{r}}_{12}(q, \theta) & \tilde{\dot{r}}_{13}(u, w, q, \theta) & 0 \\
0 & 0 & 0 & 0 \\
0 & 0 & 0 & 0 \\
0 & 0 & 0 & 0
\end{array}\right) \\
& \tilde{\dot{r}}_{12}(q, \theta)=\frac{q}{\cos ^{2} \theta} \\
& \tilde{\dot{r}}_{13}(u, w, q, \theta)=\frac{J_{y}}{m c C_{m_{\delta_{e}}}}\left(C_{L_{\delta_{e}}} \cos \alpha+C_{D_{\delta_{e}}} \sin \alpha\right) \frac{q}{\cos ^{2} \theta}
\end{aligned}
$$

\section{B. Optimal choice of the matrix function $F(\vartheta)$}

The matrix function $F(\vartheta)$ can be chosen as desired through an appropriate choice of the matrix $K(\vartheta)$, see (14), such that it is possible to assign the eigenvalues function $\lambda(\cdot): \vartheta \in \Theta \mapsto \lambda(\vartheta) \in \mathbb{R}^{4}$. By choosing a diagonal matrix function $F(\vartheta)$ :

$$
F(\vartheta)=\left(\begin{array}{cccc}
\lambda_{1}^{F}(\vartheta) & 0 & 0 & 0 \\
0 & \lambda_{2}^{F}(\vartheta) & 0 & 0 \\
0 & 0 & \lambda_{3}^{F}(\vartheta) & 0 \\
0 & 0 & 0 & \lambda_{4}^{F}(\vartheta)
\end{array}\right)
$$

some desired properties are obtained. First of all, the convergence to zero of the estimation error $\varepsilon_{R}$ when $v_{u n}=0$ and $v=0$ can be obtained by constraining $\lambda_{i}^{F}(\vartheta) \in \mathbb{C}^{-}$ $\forall \vartheta \in \Theta$. This is advantageous with respect to the case of full $F(\vartheta)$, where the stability should be proved using an LMIbased Lyapunov approach [26]. Second, the eigenvalues of $F(\vartheta)$ will correspond to $e_{1}, e_{2}, e_{3}, e_{4}$, i.e. $F(\vartheta) e_{i}=\lambda_{i}^{F}(\vartheta)$, $i=1,2,3,4$, such that it will be assured that $\varphi_{t}, \varphi_{e}$ and $\dot{\omega}_{z}$ will affect only $\varepsilon_{R, 1}, \varepsilon_{R, 2}$ and $\varepsilon_{R, 3}$, respectively, thanks to the unknown input effects decoupling discussed previously.

At this point, it remains to tune the matrix functions $\lambda_{i}^{F}(\vartheta), i=1,2,3,4$, in some optimal way. To do so, let us introduce the icing to wind/noise ratio (IWNR) for the $i$-th residual $\varepsilon_{R, i}$, as follows:

$$
I W N R_{i}(\cdot)=\frac{\left|\frac{\varepsilon_{R, i, \eta}^{\infty}}{\eta}\right|}{\left|\frac{\dot{\omega}_{x}^{M A X}}{\dot{\omega}_{x}} \varepsilon_{R, i, \omega_{x}}^{\infty}\right|+\left|\frac{\dot{\omega}_{z}^{M A X}}{\dot{\omega}_{z}} \varepsilon_{R, i, \dot{\omega}_{z}}^{\infty}\right|+\sum_{j=1}^{3}\left|\frac{v_{j}^{M A X}}{v_{j}} \varepsilon_{R, i, v_{j}}^{\infty}\right|}
$$

where $\varepsilon_{R, i, \eta}^{\infty}, \varepsilon_{R, i, \dot{\omega}_{x}}^{\infty}, \varepsilon_{R, i, \dot{\omega}_{z}}^{\infty}$ and $\varepsilon_{R, i, v_{j}}^{\infty}$ are the contributions to the steady-state value of $\varepsilon_{R, i}$ due to the icing, the wind accelerations $\dot{\omega}_{x} / \dot{\omega}_{z}$ and the $j$-th sensor noise, respectively. Notice that a scalar factor is added to weigh the contributions to $\varepsilon_{R, i}^{\infty}$ brought by the different sources of perturbation in the same way.

From the estimation error dynamics given by (18), applied to the LPV model of the UAV subject to icing/faults described in Section II, taking into account (25)-(31), and after some calculation, the following is obtained:

$$
\begin{aligned}
& \varepsilon_{R, 1, \eta}^{\infty}=-\left(\frac{\mathcal{E}_{1}(u, w, q)+\tan \theta \mathcal{E}_{2}(u, w, q)+r_{13}(u, w, \theta) \mathcal{E}_{3}(u, w, q)}{\lambda_{1}^{F}(\vartheta)}\right) \eta \\
& \varepsilon_{R, 1, \dot{\omega}_{x}}^{\infty}=\frac{\cos \theta+\sin \theta \tan \theta}{\lambda_{1}^{F}(\vartheta)} \dot{\omega}_{x} \\
& \varepsilon_{R, 1, \dot{\omega}_{z}}^{\infty}=0 \\
& \varepsilon_{R, 1, v_{1}}^{\infty}=\left(\frac{a_{11}(\cdot)+a_{21}(\cdot) \tan \theta+r_{13}(u, w, \theta) a_{31}(\cdot)}{\lambda_{1}^{F}(\vartheta)}-1\right) v_{1} \\
& \varepsilon_{R, 1, v_{2}}^{\infty}=\left(\frac{a_{12}(\cdot)+a_{22}(\cdot) \tan \theta+r_{13}(u, w, \theta) a_{32}(\cdot)}{\lambda_{1}^{F}(\vartheta)}\right) v_{2} \\
& +\left(\frac{\tilde{\dot{r}}_{12}(q, \theta)}{\lambda_{1}^{F}(\vartheta)}-\tan \theta\right) v_{2} \\
& \varepsilon_{R, 1, v_{3}}^{\infty}=\left(\frac{a_{13}(\cdot)+a_{23}(\cdot) \tan \theta+r_{13}(u, w, \theta) a_{33}(\cdot)}{\lambda_{1}^{F}(\vartheta)}\right) v_{3} \\
& +\left(\frac{\tilde{r}_{13}(u, w, q, \theta)}{\lambda_{1}^{F}(\vartheta)}-r_{13}(u, w, \theta)\right) v_{3} \\
& \varepsilon_{R, 2, \eta}^{\infty}=-\frac{r_{23}}{\lambda_{2}^{F}(\vartheta)} \mathcal{E}_{3}(u, w, q) \eta \\
& \varepsilon_{R, 2, \dot{\omega}_{x}}^{\infty}=0 \\
& \varepsilon_{R, 2, \omega_{z}}^{\infty}=0 \\
& \varepsilon_{R, 2, v_{1}}^{\infty}=\frac{r_{23} a_{31}(\cdot)}{\lambda_{2}^{F}(\vartheta)} v_{1} \\
& \varepsilon_{R, 2, v_{2}}^{\infty}=\frac{r_{23} a_{32}(\cdot)}{\lambda_{2}^{F}(\vartheta)} v_{2} \\
& \varepsilon_{R, 2, v_{3}}^{\infty}=\left(\frac{r_{23} a_{33}(\cdot)}{\lambda_{2}^{F}(\vartheta)}-r_{23}\right) v_{3} \\
& \varepsilon_{R, 3, \eta}^{\infty}=-\left(\frac{\mathcal{E}_{2}(u, w, q)+r_{33}(u, w) \mathcal{E}_{3}(u, w, q)}{\lambda_{3}^{F}(\vartheta)}\right) \eta \\
& \varepsilon_{R, 3, \omega_{x}}^{\infty}=\frac{\sin \theta}{\lambda_{3}^{F}(\vartheta)} \dot{\omega}_{x} \\
& \varepsilon_{R, 3, \dot{\omega}_{z}}^{\infty}=-\frac{\cos \theta}{\lambda_{3}^{F}(\vartheta)} \dot{\omega}_{z} \\
& \varepsilon_{R, 3, v_{1}}^{\infty}=\left(\frac{a_{21}(\cdot)+r_{33}(u, w) a_{31}(\cdot)}{\lambda_{3}^{F}(\vartheta)}\right) v_{1} \\
& \varepsilon_{R, 3, v_{2}}^{\infty}=\left(\frac{a_{22}(\cdot)+r_{33}(u, w) a_{32}(\cdot)}{\lambda_{3}^{F}(\vartheta)}-1\right) v_{2} \\
& \varepsilon_{R, 3, v_{3}}^{\infty}=\left(\frac{a_{23}(\cdot)+r_{33}(u, w) a_{33}(\cdot)}{\lambda_{3}^{F}(\vartheta)}-r_{33}(u, w)\right) v_{3}
\end{aligned}
$$

Hence, it is evident that the icing to wind/noise ratio for the $i$-th residual, defined in (32), depends on $\vartheta$ due to the eigenvalue $\lambda_{i}(\vartheta)$ and the presence of the state variables, 
which are included in $\vartheta$. In order to enhance the residual's ability to reject the wind acceleration disturbance and the noise, and to increase the sensitivity to the icing, an optimal choice of $\lambda_{i}^{F}(\vartheta)$ would be the one that maximizes the corresponding icing to wind/noise ratio $^{2}$, i.e.

$$
\lambda_{i, O P T}^{F}(\vartheta)=\arg \max _{\lambda_{i}^{F}(\vartheta)<0} I W N R_{i}\left(\vartheta, \lambda_{i}^{F}(\vartheta)\right)
$$

The optimization problem (51) corresponds to an infinite number of constraints, which can be reduced to a finite number by gridding the set $\Theta$, and calculating (51) for each point of this grid. Then, for a given value of $\vartheta$, the matrix $F(\vartheta)$ would be calculated as a weighted convex combination of the nearby gridding points. In this way, the convergence to zero of the estimation error $\varepsilon_{R}$ when $v_{u n}=0$ and $v=0$ would still be guaranteed, due to the fact that a convex combination of negative definite diagonal matrices would preserve the property of being negative definite diagonal. On the other hand, even though the optimality of the eigenvalues with respect to the icing to wind/noise ratio would be guaranteed only at the gridding points, it is reasonable that if the grid is sufficiently dense, the sub-optimality for values of $\vartheta$ different from the grid ones would be only a slight degradation with respect to the optimality.

\section{Fault/icing diagnosis algorithm}

The following algorithm is proposed to decide about the occurrence of faults and icing.

\section{Decision Algorithm.}

$$
\begin{aligned}
& \text { if }\left|\varepsilon_{R, 1}(t)\right| \leq \varepsilon_{R, 1}^{t h} \wedge\left|\varepsilon_{R, 2}(t)\right| \leq \varepsilon_{R, 2}^{t h} \wedge\left|\varepsilon_{R, 3}(t)\right| \leq \varepsilon_{R, 3}^{t h} \\
& \text { then "no faults/no icing" } \\
& \text { if }\left|\varepsilon_{R, 1}(t)\right|>\varepsilon_{R, 1}^{t h} \wedge\left|\varepsilon_{R, 2}(t)\right| \leq \varepsilon_{R, 2}^{t h} \wedge\left|\varepsilon_{R, 3}(t)\right| \leq \varepsilon_{R, 3}^{t h} \\
& \quad \text { then "fault in thrust" } \\
& \text { if }\left|\varepsilon_{R, 1}(t)\right| \leq \varepsilon_{R, 1}^{t h} \wedge\left|\varepsilon_{R, 2}(t)\right|>\varepsilon_{R, 2}^{t h} \wedge\left|\varepsilon_{R, 3}(t)\right| \leq \varepsilon_{R, 3}^{t h} \\
& \text { then "fault in elevator" } \\
& \text { else "possible icing" }
\end{aligned}
$$

The thresholds $\varepsilon_{R, 1}^{t h}, \varepsilon_{R, 2}^{t h}, \varepsilon_{R, 3}^{t h}$ should be calculated in such a way that the residuals never exceed them due to the wind turbulence.

\section{CASE STUdy}

Let us consider the case study of a small UAV (Zagi Flying Wing, see Table II for the parameters appearing in the quasiLPV model described in Section II) flying at an altitude $h=150 \mathrm{~m}$ and subject to wind accelerations calculated using the Dryden model provided by the Aerospace Toolbox of MATLAB, with a light probability of exceedance of highaltitude intensity.

The aircraft is controlled by an autopilot, responsible of maintaining the horizontal velocity $u$ around the desired

\footnotetext{
${ }^{2}$ Notice that the proposed approach is akin to the $\mathcal{H} / \mathcal{H}_{\infty}$ fault detection observer design described in [27].
}

TABLE II

SYSTEM PARAMETERS VALUES

\begin{tabular}{cccccc}
\hline Param. & Value & Param. & Value & Param. & Value \\
\hline$m$ & $1.56 \mathrm{~kg}$ & $C_{L_{0}}$ & 0.09167 & $C_{D_{q}}$ & 0 \\
$J_{y}$ & $0.0576 \mathrm{~kg} \mathrm{~m}^{2}$ & $C_{D_{0}}$ & 0.01631 & $C_{m_{q}}$ & -1.3990 \\
$S$ & $0.2589 \mathrm{~m}^{2}$ & $C_{m_{0}}$ & -0.02338 & $C_{L_{\delta_{e}}}$ & 0.2724 \\
$c$ & $0.3302 \mathrm{~m}$ & $C_{L_{\alpha}}$ & 3.5016 & $C_{D_{\delta_{e}}}$ & 0.3045 \\
$S_{\text {prop }}$ & $0.0314 \mathrm{~m}^{2}$ & $C_{D_{\alpha}}$ & 0.2108 & $C_{m_{\delta_{e}}}$ & -0.3254 \\
$\rho$ & $1.2682 \mathrm{~kg} / \mathrm{m}^{3}$ & $C_{m_{\alpha}}$ & -0.5675 & $C_{\text {prop }}$ & 1.0 \\
$k_{m}$ & 20 & $C_{L_{q}}$ & 2.8932 & & \\
\hline
\end{tabular}

value $u_{r e f}=20 \mathrm{~m} / \mathrm{s}$ with the following reference pitch angle:

$$
\theta_{r e f}=\left\{\begin{array}{cc}
0 & t \leq 100 s \\
\frac{(t-100)}{1500} & 100 s<t \leq 250 s \\
\frac{(450-t)}{2000} & 250 s<t \leq 450 s \\
0 & t>450 s
\end{array}\right.
$$

It is assumed that the noise affecting the sensor measurements in (13) is uniformly distributed within the following intervals: $v_{1} \in[-1,1], v_{2} \in[-1,1], v_{3} \in[-0.001,0.001]$, $v_{4} \in[-0.008,0.012]$.

Three different fault scenarios have been considered, as follows:

\section{A. Fault scenario 1 (FS1)}

The effective thrust input $\delta_{t}$ is subject to a loss of efficiency with respect to its nominal value $\delta_{t}^{*}$ :

$$
\delta_{t}(t)=0.7 \delta_{t}^{*}(t)
$$

The fault is linearly incipient, such that the loss of efficiency starts at time $t=200 \mathrm{~s}$ and equals (53) from time $t=210 \mathrm{~s}$.

\section{B. Fault scenario 2 (FS2)}

The effective elevator deflection $\delta_{e}$ is subject to a loss of efficiency with respect to its nominal value $\delta_{e}^{*}$ :

$$
\delta_{e}(t)=0.9 \delta_{e}^{*}(t)
$$

The fault is linearly incipient, such that the loss of efficiency starts at time $t=200 \mathrm{~s}$ and equals (54) starting from time $t=210 s$.

\section{Fault scenario 3 (FS3)}

The aircraft is subject to icing, modeled as in (6)-(9), taking into account the coefficients $K_{i}$ listed in Table III. The icing starts at time $t=200 \mathrm{~s}$ and slowly increases $\eta$ from 0 to 0.2 , such that $\eta=0.2$ starting from time $t=400 \mathrm{~s}$.

The optimization problem (51) has been solved in 1296 gridding points, that correspond to the partition of each interval of variation of the state variables $(u \in[15,25]$, $w \in[1,5], q \in[-0.001,0.001], \theta \in[-0.01,0.01])$ in 5 subintervals, providing the optimal values for the eigenvalues $\lambda_{i}^{F}(\vartheta), i=1,2,3$, that are shown in Fig. 1 (due to the difference in order of magnitude of the optimal values for the eigenvalues, a logarithmic scale is used for presentation purpose).

Remark: Notice that the optimization of $F(\vartheta)$, which is the most computationally demanding part of the proposed 
TABLE III

COEFFICIENTS $K_{i}$ FOR AN ALL ICED CONFIGURATION

\begin{tabular}{cccccc}
\hline Coeff. & Value & Coeff. & Value & Coeff. & Value \\
\hline$K_{L_{0}}$ & 0 & $K_{L_{\alpha}}$ & -0.5000 & $K_{L_{q}}$ & -0.0675 \\
$K_{L_{\delta_{e}}}$ & -0.4770 & $K_{D_{0}}$ & 2.5610 & $K_{D_{\alpha}}$ & 0 \\
$K_{D_{q}}$ & 0 & $K_{D_{\delta_{e}}}$ & 0 & $K_{m_{0}}$ & 0 \\
$K_{m_{\alpha}}$ & -0.4960 & $K_{m_{q}}$ & -0.1755 & $K_{m_{\delta_{e}}}$ & -0.5000 \\
\hline
\end{tabular}

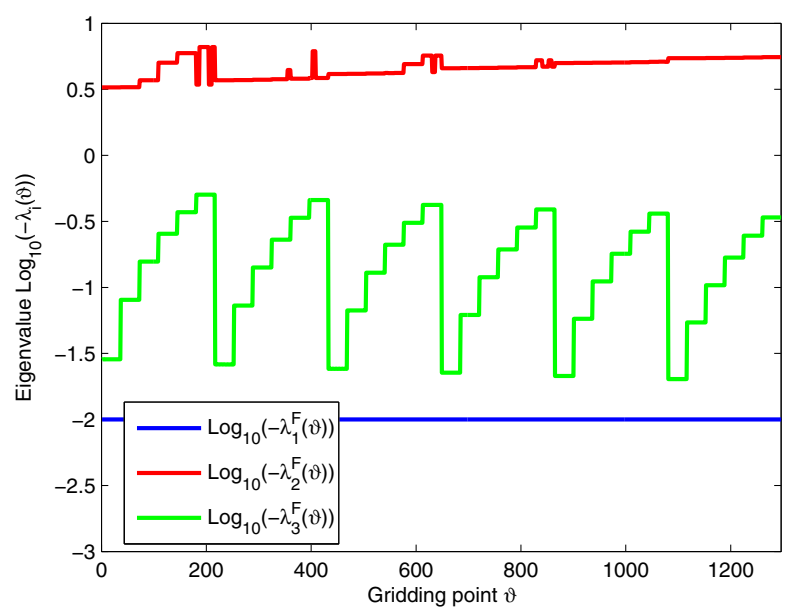

Fig. 1. Optimal eigenvalues at the gridding points (logarithmic scale).

strategy, is performed offline. Only the weighted combination of the matrices defined at the gridding points is performed online, taking into account the value of the varying parameter vector.

Figs. 2-4 show the residuals obtained in the considered fault scenarios using a proportional UIO, as described in [16]. On the other hand, Figs. 5-7 show the evolution of the residuals obtained using the proposed PI-UIO. From a qualitative comparison between the results, it is evident that the latter exhibits a stronger rejection of the undesired effects, i.e. sensor noise and wind acceleration disturbance.

It has been noticed that the frequency content of the residuals $\varepsilon_{R, i}(t), i=1,2,3$, changes under faults/icing occurrence. In fact, in absence of faults/icing, the residuals are excited only by the wind acceleration, which is mostly a high frequency disturbance. On the other hand, the actuator faults and icing effects increase the low frequency content of the variables $\varepsilon_{R, i}(t)$. Thus, low-pass filtering the residuals indicates more clearly the presence of faults/icing. More specifically, the low-pass filtered residuals are obtained as follows:

$$
\dot{\phi}_{R, i}(t)=-a_{i} \phi_{R, i}(t)+a_{i} \varepsilon_{R, i}(t) \quad i=1,2,3
$$

where $a_{i}$ is a design parameter that should be chosen to guarantee that only the part of the frequency spectrum of $\varepsilon_{R, i}(t)$ affected by faults/icing is preserved. Then, the decision algorithm proposed in the previous section is slightly modified by replacing all instances of $\varepsilon_{R, i}$ with $\phi_{R, i}, i=$ $1,2,3$.

Figs. 8-10 show the evolution of the low-pass filtered

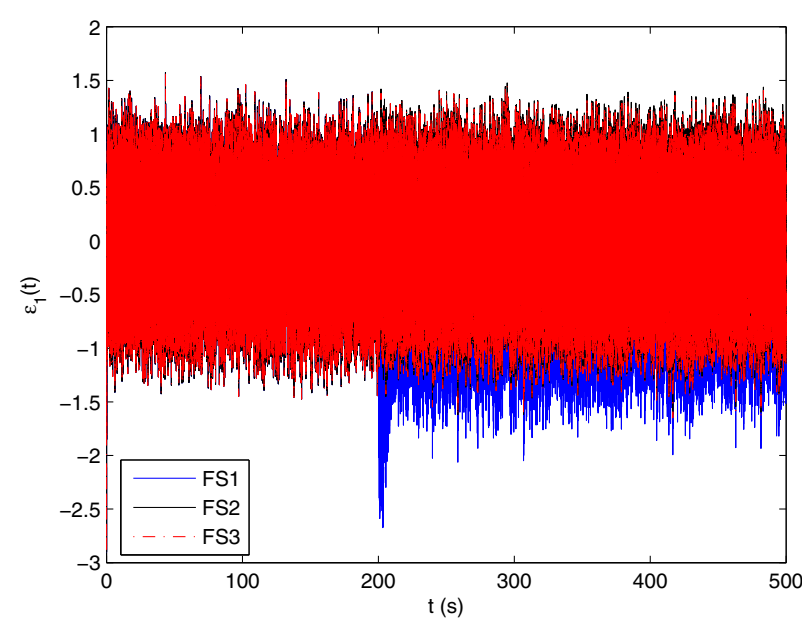

Fig. 2. Residual $\varepsilon_{1}(t)$ using a proportional UIO.

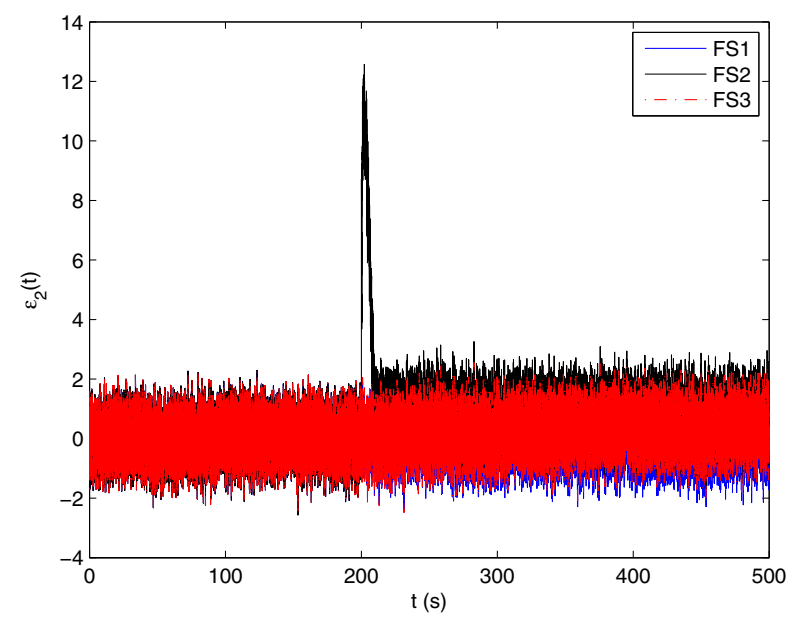

Fig. 3. Residual $\varepsilon_{2}(t)$ using a proportional UIO.

residuals, obtained using the proposed PI-UIO and (55) with $a_{i}=0.01$, in the considered fault scenarios.

Concerning the fault/icing diagnosis, it can be seen that in fault scenario 1 (fault in the thrust), the only residual that differs considerably from 0 after $t=200 \mathrm{~s}$ is $\phi_{R, 1}(t)$. On the other hand, in fault scenario 2 (fault in the elevator), the residual which exhibits an increase of its value after $t=200 \mathrm{~s}$ is $\phi_{R, 2}$. Finally, under icing occurrence all the residuals increase their values considerably. According to the decision algorithm proposed in the previous section, once appropriate thresholds for the residuals have been chosen, the occurrence of faults/icing can be detected and isolated successfully, proving the effectiveness of the proposed approach.

\section{CONCLUSIONS}

This paper has proposed a linear parameter varying (LPV) proportional integral unknown input observer (PI-UIO) for fault/icing detection in unmanned aerial vehicles (UAVs). The advantage of the proposed LPV PI-UIO is that it can 


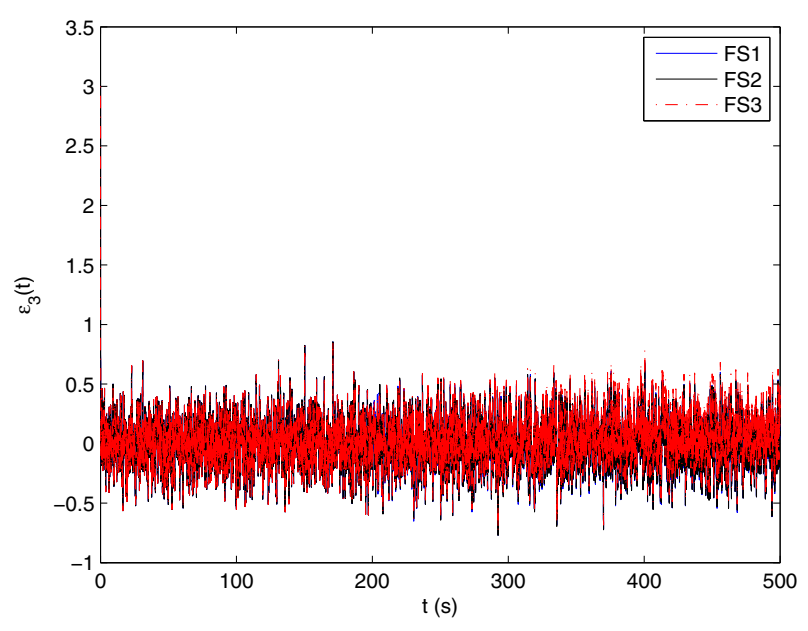

Fig. 4. Residual $\varepsilon_{3}(t)$ using a proportional UIO.

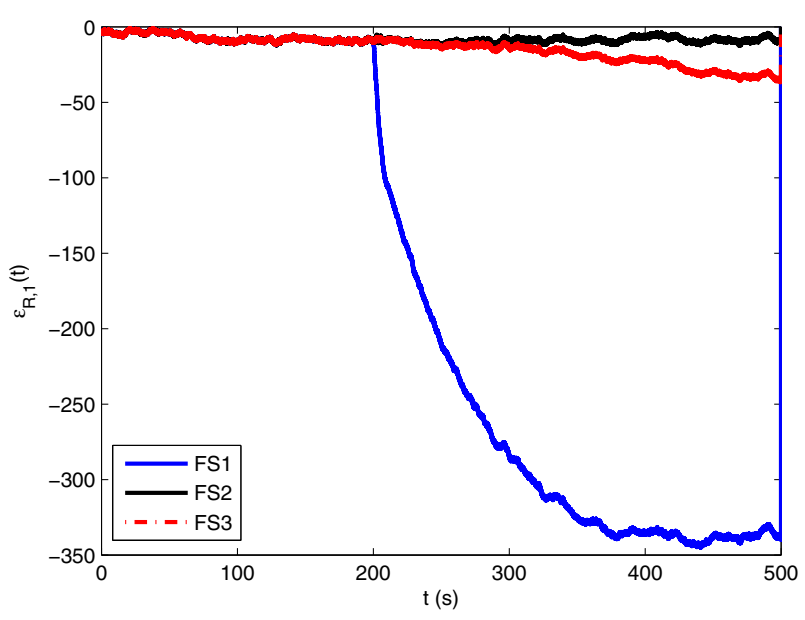

Fig. 5. Residual $\varepsilon_{R, 1}(t)$ using the proposed proportional integral UIO.

take into account operating point variations in an elegant way using the LPV paradigm. Moreover, the presence of the integral term avoids the appearance of the noise derivative term in the estimation error equation, thus increasing the noise rejection properties. It has been shown how an optimal design of the PI-UIO may be performed by increasing the sensitivity to icing, while decreasing the sensitivity to wind acceleration disturbance and noise, through the introduction of the icing to wind/noise ratio (IWNR).

The case study of a Zagi Flying Wing UAV has shown the effectiveness of the decision algorithm, which identifies correctly unexpected changes in the system dynamics due to actuator faults or icing, using low-pass filtered residuals obtained from the proposed PI-UIO.

Future research will extend the results to a 9-DOF aircraft model, and will include parametric uncertainty in the model, in order to perform a robust fault/icing diagnosis.

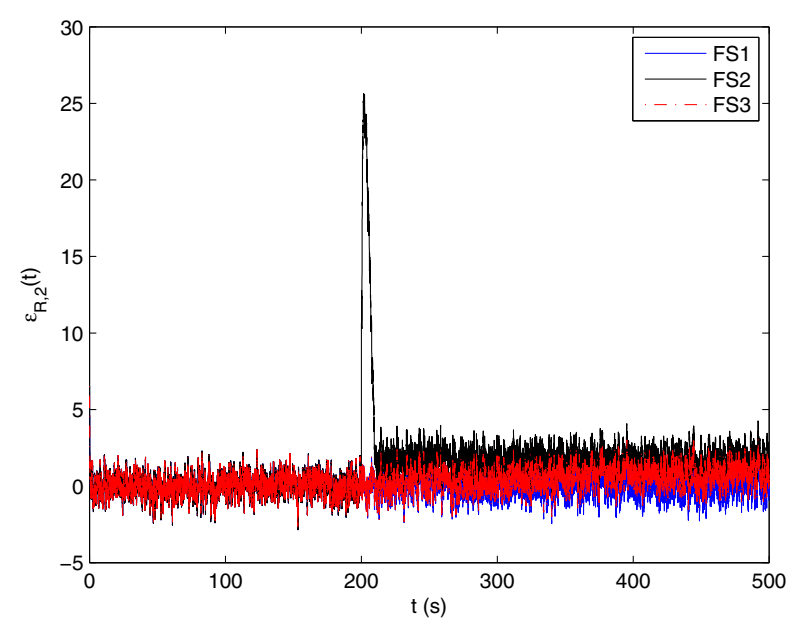

Fig. 6. Residual $\varepsilon_{R, 2}(t)$ using the proposed proportional integral UIO.

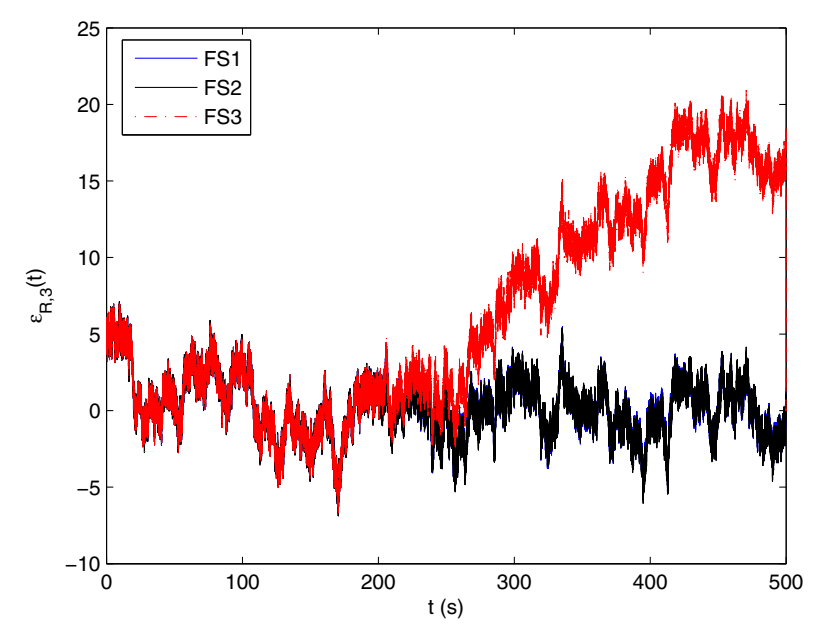

Fig. 7. Residual $\varepsilon_{R, 3}(t)$ using the proposed proportional integral UIO.

\section{REFERENCES}

[1] C. De Persis and A. Isidori, "A geometric approach to nonlinear fault detection and isolation," IEEE Transactions on Automatic Control, vol. 46, no. 6, pp. 853-865, 2001.

[2] C. De Persis, R. De Santis, and A. Isidori, "Nonlinear actuator fault detection and isolation for a VTOL aircraft," in Proceedings of the American Control Conference, 2001, pp. 4449-4454.

[3] P. Kaboré, S. Othmar, T. F. McKenna, and H. Hammouri, "Observerbased fault diagnosis for a class of non-linear systems - Application to a free radical copolymerization," International Journal of Control, vol. 73, no. 9, pp. 787-803, 2000.

[4] P. Kaboré and H. Wang, "Design of fault diagnosis filters and faulttolerant control for a class of nonlinear systems," IEEE Transactions on Automatic Control, vol. 46, no. 11, pp. 1805-1810, 2001.

[5] F. Caliskan and C. Hajijev, "A review of in-flight detection and identification of aircraft icing and reconfigurable control," Progress in Aerospace Sciences, vol. 60, pp. 12-34, 2013.

[6] R. Gent, N. Dart, and J. Cansdale, "Aircraft icing," Phil. Trans. of the Royal Soc. of London. Series A: Mathematical, Physical and Engineering Sciences, vol. 358, pp. 2873-2911, 2000.

[7] J. W. Elliott and F. T. Smith, "Ice formation on a smooth or rough cold surface due to the impact of a supercooled water droplet," Journal of Engineering Mathematics, pp. 1-30, 2015. 


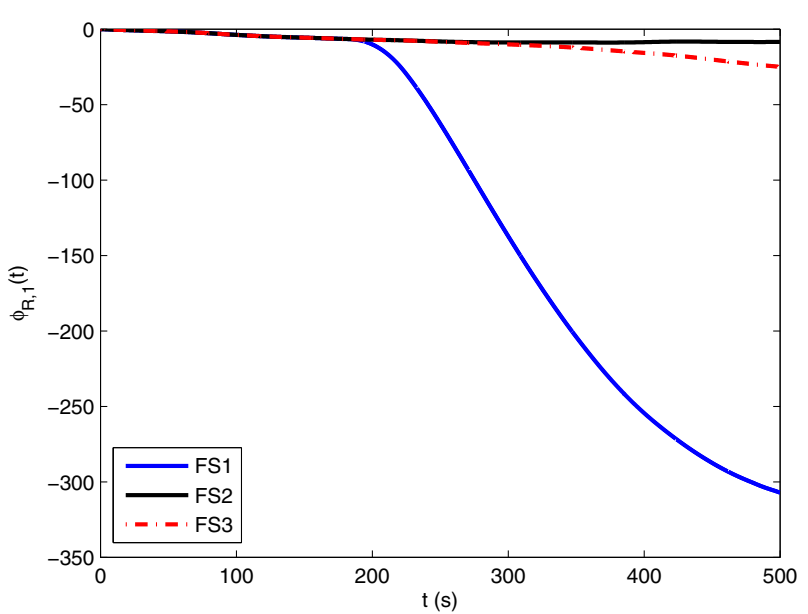

Fig. 8. Low-pass filtered residual $\phi_{R, 1}(t)$ using the proposed PI-UIO.

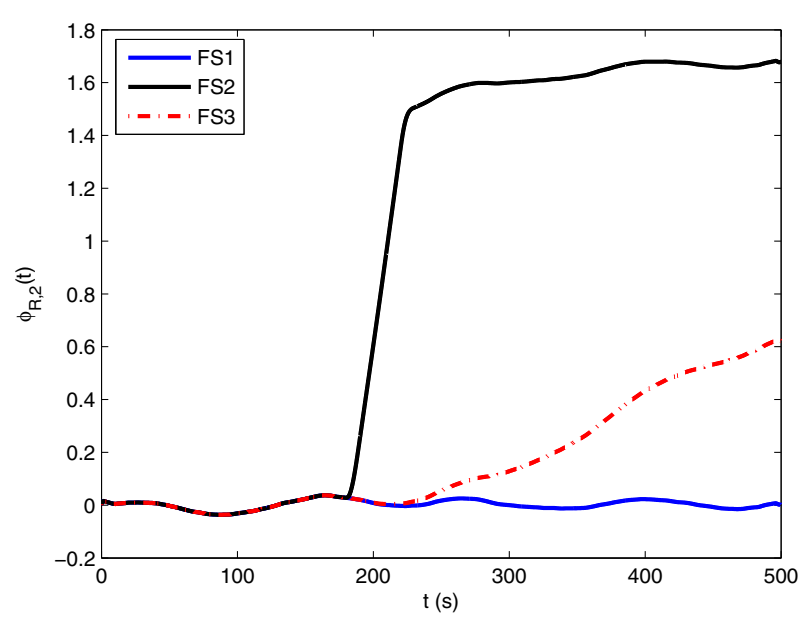

Fig. 9. Low-pass filtered residual $\phi_{R, 2}(t)$ using the proposed PI-UIO.

[8] M. B. Bragg, A. P. Broeren, and L. Blumenthal, "Iced-airfoil aerodynamics," Progress in Aerospace Sciences, vol. 41, no. 5, pp. 323-362, 2005.

[9] K. L. Sørensen, A. S. Helland, and T. A. Johansen, "Carbon nanomaterial-based wing temperature control system for in-flight antiicing and de-icing of unmanned aerial vehicles," in Proceedings of the IEEE Aerospace Conference, 2015.

[10] C. Hajijev and F. Caliskan, Fault diagnosis and reconfiguration in flight control systems. Springer Science and Business Media, 2003.

[11] M. Tousi and K. Khorasani, "Robust observer-based fault diagnosis for an unmanned aerial vehicle," in Proceedings of the IEEE International Systems Conference (SysCon), 2011, pp. 428-434.

[12] A. Cristofaro and T. A. Johansen, "An unknown input observer approach to icing detection for unmanned aerial vehicles with linearized longitudinal motion," in American Control Conference, 2015.

[13] M. M. Seron, T. A. Johansen, J. A. De Doná, and A. Cristofaro, "Detection and estimation of icing in unmanned aerial vehicles using a bank of unknown input observers," in Proceedings of the 5th Australian Control Conference (UACC), 2015, pp. 87-92.

[14] A. Cristofaro and T. A. Johansen, "Icing detection and identification for unmanned aerial vehicles: multiple model adaptive estimation," in European Control Conference, 2015.

[15] K. L. Sørensen, M. Blanke, and T. A. Johansen, "Diagnosis of wind icing through lift and drag coefficient change detection for small unmanned aircraft," in Proc. 9th IFAC Symposium on Fault Detection,

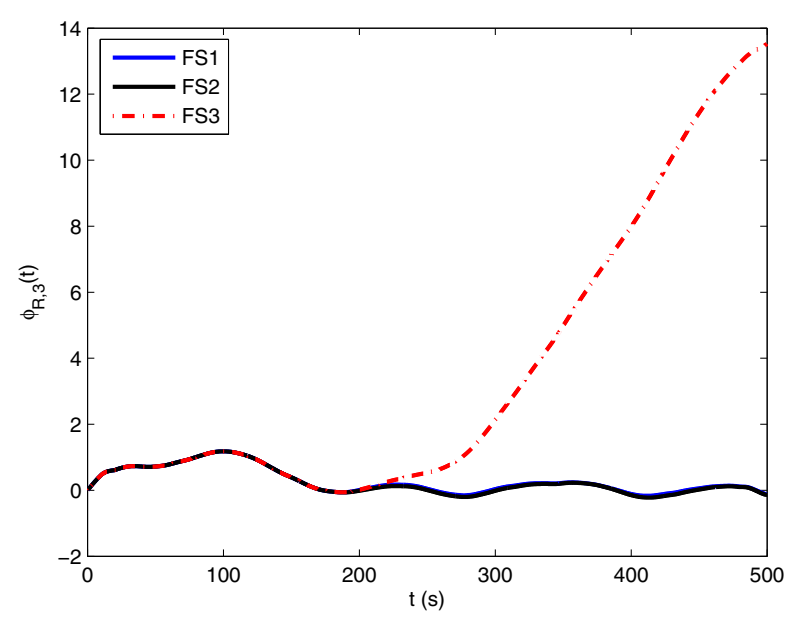

Fig. 10. Low-pass filtered residual $\phi_{R, 3}(t)$ using the proposed PI-UIO.

Supervision and Safety of Technical Processes, 2015.

[16] D. Rotondo, A. Cristofaro, T. A. Johansen, F. Nejjari, and V. Puig, "Icing detection in unmanned aerial vehicles with longitudinal motion using an LPV unknown input observer," in 9th IEEE Multi-Conference on Systems and Control, 2015.

[17] K. K. Busawon and P. Kabore, "On the design of integral and proportional integral observers," in Proceedings of the American Control Conference, 2000, pp. 3725-3729.

[18] D. Koenig and S. Mammar, "Design of proportional-integral observers for unknown input descriptor systems," IEEE Transactions on Automatic Control, vol. 47, no. 12, pp. 2057-2062, 2002.

[19] D. Koenig, "Unknown input proportional multiple-integral observer design for linear descriptor systems: application to state and fault estimation," IEEE Transactions on Automatic Control, vol. 50, no. 2, pp. 212-217, 2005.

[20] T. Youssef, M. Chadli, H. R. Karimi, and M. Zelmat, "Design of unknown inputs proportional integral observers for TS fuzzy models," Neurocomputing, vol. 123, pp. 156-165, 2014.

[21] R. W. Beard and T. W. McLain, Small Unmanned Aircraft: Theory and Practice. Princeton, NJ, USA: Princeton University Press, 2012.

[22] A. Kwiatkowski, M.-T. Boll, and H. Werner, "Automated Generation and Assessment of Affine LPV Models," in Proceedings of the 45th IEEE Conference on Decision and Control, 2006, pp. 6690-6695.

[23] D. Rotondo, V. Puig, F. Nejjari, and M. Witczak, "Automated generation and comparison of Takagi-Sugeno and polytopic quasi-LPV models," Fuzzy Sets and Systems, vol. in press, 2015.

[24] F. M. Hoblit, Gust loads on aircraft: concept and applications. Washington, DC, USA: American Institute of Aeronautics and Astronautics, 1988.

[25] M. B. Bragg, T. Hutchinson, J. Merret, R. Oltman, and D. Pokhariyal, "Effect of ice accretion on aircraft flight dynamics," in Proceedings of the 38th AIAA Aerospace Science Meeting and Exhibit, 2000.

[26] G. Becker, A. Packard, D. Philbrick, and G. Balas, "Control of parametrically-dependent linear systems: a single quadratic Lyapunov approach," in Proceedings of the American Control Conference, 1993, pp. 2795-2799.

[27] J. L. Wang, G.-H. Yang, and J. Liu, "An LMI approach to $\mathcal{H} / \mathcal{H}_{\infty}$ fault detection observer design," Automatica, vol. 43, pp. 1656-1665, 2007. 